\title{
Gas-Dynamic Analysis of Processes in a Small-Sizes Two-Stroke Combustion Engine
}

\author{
V.V. Biryuk, A.A. Gorshkalev, S.S. Kayukov and D.A. Uglanov*
}

Samara State Aerospace University, Samara, Russia

\begin{abstract}
The paper presents a procedure for gas-dynamic analysis of processes in the air-gas channel of a small-sized two-stroke combustion engine. All steps are described. At the first stage a three-dimensional model of the interior space of the air-gas channel of a piston small-power engine was built; this model was split into nine different volumes according to the methodology of volumes partition for different kinds of grids application, which is provided in source materials. At the second stage all required sizes and types of finite elements were set for the correct operation of functions of resplitting and layer-by-layer re-formation of a grid in the cylinder volume and in the sub piston area. At the third stage all the necessary settings in the CFD-code ANSYS Fluent were made. Such as a transient simulation method, a turbulence model, parameters of mixture formation and fuel evaporation process, setup of intersection zones of the grid, setup of permeability of the boundaries between the calculated areas, setup of grid re-formation parameters, motion parameters of the piston and other moving elements of the simulated engine were introduced, setup of parameters and characteristics of the fuel injection process. User Defined Function (UDF) was used to set the rotation of the crankshaft. The calculations found fields of distribution of the basic parameters in the air-gas channel of the small-sized combustion engine, such as pressure, temperature, speed and composition of the working body. According to the simulation results power and fuel efficiency of the small-sized enginewere calculated.
\end{abstract}

Keywords: ANSYS, boundary conditions, CAD/CAE-systems, combustion engine, combustion engine engineering, fluent, grid of finite-elements.

\section{INTRODUCTION}

Currently, during combustion engines engineering minimum expenses and time must be used. The efficient instruments that can be used to solve the problem of analysis and building of combustion engines are modern CAD systems (CAD).

The technique of modeling of thermal processes in an air model small-power combustion engine is using $\mathrm{CAD} / \mathrm{CAE}$ technologies was presented. The solution to this problem is one of the most important tasks in the field of combustion engines engineering. The purpose of the thermal processes modeling is visual representation of the operating principle of an air model two-stroke engine through its three-dimensional model. Simulation of thermal processes in the combustion engine is performed using ANSYS Fluent CFD-code [1]. The efficiency of the combustion engine depends on a combination of complex processes, namely: fuel components supply, airand-fuel mixture preparation, fuel combustion and combustion gases discharge, etc. The studies conducted using CFDmodeling at the early stages of the engine designing help us to optimize each of these processes. The complexity of the modeling process is associated with the analysis grid model building, which should move in line with the movement of the working bodyinside the piston engine cylinder [2].
This technique can be used to obtain the fields of distribution of the main thermodynamic parameters in the piston-cylinder group of combustion engines with the help of ANSYS Fluent program, and allows to gets kills of working with moving analysis grids.

\section{METHOD}

At the first stage a three-dimensional model of the smallsized combustion engine was built using Solid Works CADeditor. Then with the help of volumes subtraction the internal volume of the air-gas channel of the engine was built. The air-gas channel of the engine was split into nine volumes, according to the authors' method of three-dimensional models preparation for grid elements application. As the result a three-dimensional model ready for creation of a grid model presented in Fig. (1) was built.

Creation of a grid model is an integral part of computer aided engineering (CAE). Grid quality affects the accuracy, convergence and speed of decision receiving. Furthermore, it usually takes a significant portion of the total time of execution of computer engineering evaluation. Therefore, qualitative and more automated grid generation tools give better results.

Grid generation was performed using ANSYS Meshing grid generator based of Workbench platform. When setting the grid parameters the range of grid element sizes was selected to be 1 to $1.5 \mathrm{~mm}$, the gradient of grid elements growth-equal to 1.05 and the type of a finite elementtetrahedron. 


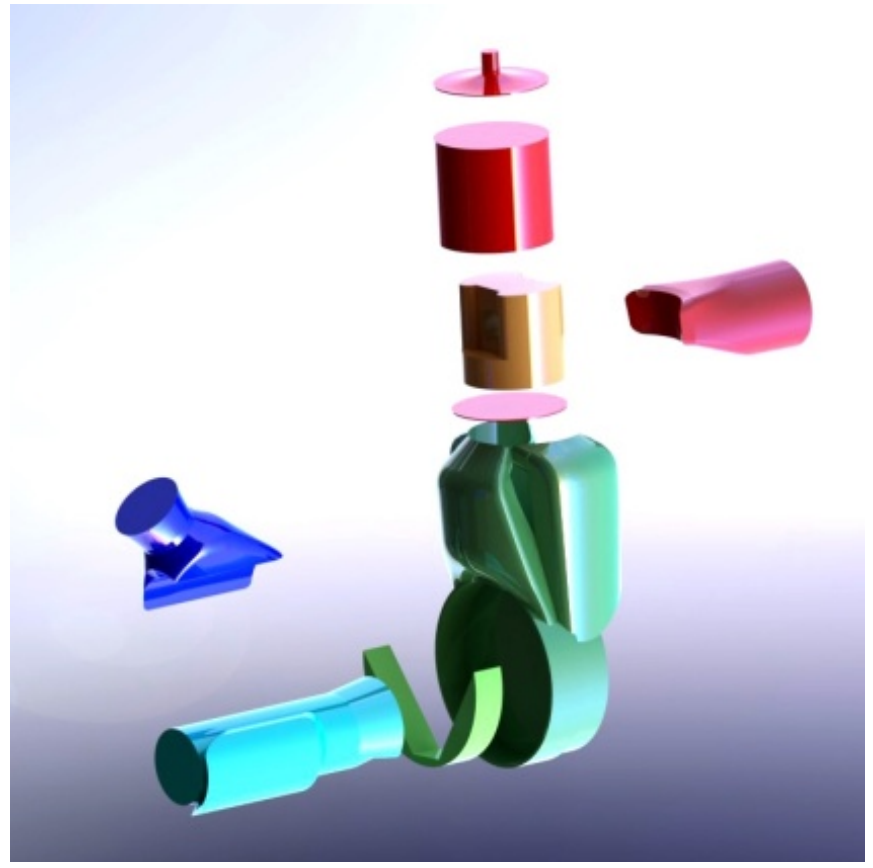

Fig. (1). Model splitting scheme.

For correct operation of layer-by-layer re-construction of the grid during the calculation the size of the grid element was set to $0.5 \mathrm{~mm}$, the finite element type was set to hexahedron and the method of grid partitioning in the cylinder volume and the sub piston area was set. As the result of the construction the grid model presented in Fig. (2) was obtained. Boundary surfaces of the entrance to the airgas channel and exit of the working body from it were set Names and boundary penetrability conditions are automatically assigned to matching surfaces of different volumes (Interface).

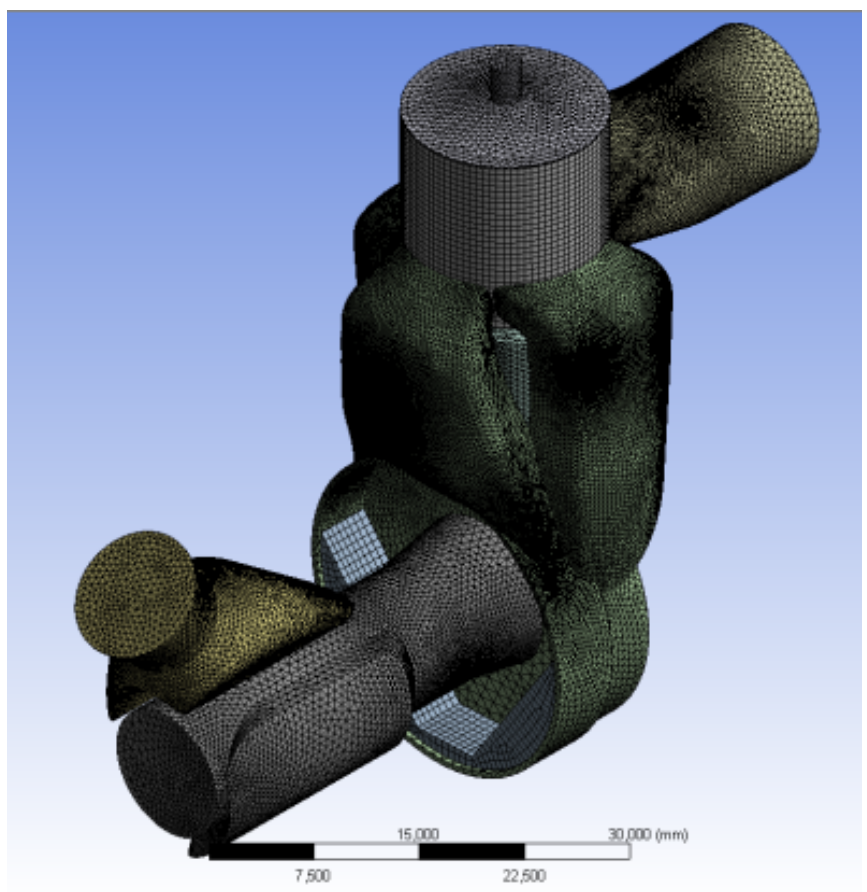

Fig. (2). The grid model of the engine air-gaschannel.

ANSYS Fluent was used to calculate the gas-dynamic and thermal processes in the air-gas channel of the combustion engine. Transient simulation method considering turbulent flows was chosen to solve this problem [3]. Appropriate for this task model of turbulence k- $\varepsilon$ was chosen to ensure high accuracy of the calculations. It is a simple two-parameter model of turbulence where two transfer equations determining turbulent velocity and vortex decay time are solved. In solving this problem heat exchange and heat transfer was taken into account by inclusion of an energy equation.

At the next stage of modeling parameters of chemicals mixing were specified. Since the fuel used in the engine is liquid parameters of fuel evaporation were set. Droplet Collision and Droplet Breakup calculation was set up. In the boundary conditions menu the necessary parameters on the boundaries of the model were set. Further zones of grid intersection were set up. This setting is necessary to obtain a moving grid and to set up the permeability of the boundaries between the calculated areas [4].

After that, grid motion parameters were set up and a dynamic model of grid motion and its main parameters were set. Parameters for grid motion plan were specified in options.

In the build-in option in-Cylinder combustion engine parameters, such as engine speed, initial turning angle of the crank shaft at which the calculation starts, time of one cycle in degrees (for a two-stroke engine $360^{\circ}$ ), turning angle of the crank shaft per one stem, piston stroke and crank length were specified [5].

The next stage of the simulation was to develop and load the file for compilation of UD Frotation function of the crankshaft, the text of which is provided below:

\#include "udf.h"

DEFINE_CG_MOTION (crank, dt, vel, omega, time, dtime)

\{Domain * domain;

domain $=$ Get_Domain (1);

omega [2] $=418.8667 ;\}$

After that motion parameters of the piston and other moving elements of the simulated engine were introduced in accordance with the following Table $\mathbf{1}$.

\section{RESULTS}

The calculations found the fields of distribution of the basic parameters in the air-gas channel of the small-size combustion engine, such as pressure, temperature, speed and composition of the working body. According to the simulation results power and fuel efficiency of small-size engine were calculated.

The results of the calculation were saved as an animation of the distribution fields of the corresponding thermodynamic parameters. The figures show some of them. The field of distribution of pressures and velocity vectors in the longitudinal section are shown in Figs. $(\mathbf{3}, \mathbf{4})$.

As a conclusion, it is worth noting that the method of small-sized two-stroke combustion engine model calculation was developed, using which it will be possible to calculate a model of an engine with a resonance tube taking into account acoustic effects. 
Table 1. Motion parameters of various zones.

\begin{tabular}{|c|c|c|c|c|c|c|}
\hline Zone Names & Type & Motion UDF/Profile & $\mathbf{X}$ & $\mathbf{Y}$ & $\mathbf{Z}$ & Meshing Options (m) \\
\hline int_kv1_kar1_kv & RigidBody & crank::libudf & & & & 0,0005 \\
\hline int_kv1_kar1-kv-prot & RigidBody & crank::libudf & & & & 0,0005 \\
\hline int_kv_vh & RigidBody & crank::libudf & & & & 0,0005 \\
\hline int_plosk_pod-porsh & RigidBody & $* *$ piston-full ${ }^{* *}$ & 0 & 1 & 0 & 0,0005 \\
\hline interior_kv & RigidBody & crank::libudf & & & & 0,0005 \\
\hline inter_kv-prot & RigidBody & crank::libudf & & & & 0,0005 \\
\hline wall-kv-prot & RigidBody & crank::libudf & & & & 0,0005 \\
\hline
\end{tabular}

$1.09 e+05$
$1.09 e+05$
$1.08 e+05$
$1.08 e+05$
$1.07 e+05$
$1.07 e+05$
$1.07 e+05$
$1.06 e+05$
$1.06 e+05$
$1.06 e+05$
$1.05 e+05$
$1.05 e+05$
$1.04 e+05$
$1.04 e+05$
$1.04 e+05$
$1.03 e+05$
$1.03 e+05$
$1.02 e+05$
$1.02 e+05$
$1.02 e+05$
$1.01 e+05$

\section{Contours of Total Pressure (pascal) $($ Time $=3.0000 \mathrm{e}-03$ )} Crank Angle $=540.00(\mathrm{deg})$

Fig. (3). Field of distribution of pressures in the longitudinal section. 


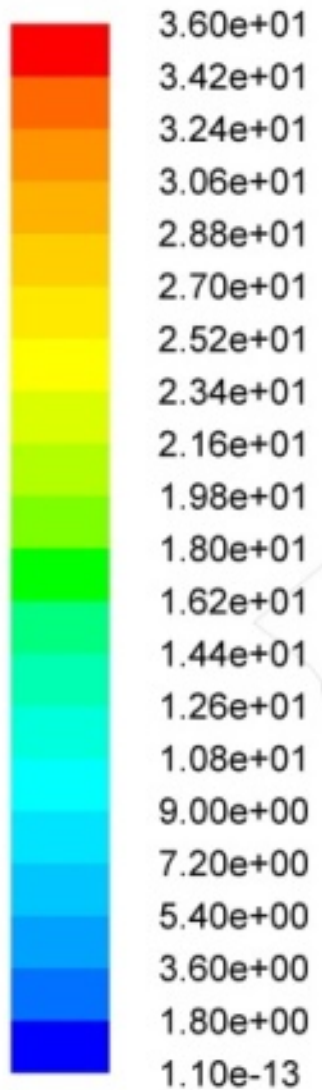

$3.60 \mathrm{e}+01$

$3.42 e+01$

$3.06 \mathrm{e}+01$

$2.88 \mathrm{e}+01$

$2.70 \mathrm{e}+01$

$2 \mathrm{e}+01$

$34 \mathrm{e}+01$

$\mathrm{e}+01$

$1.62 \mathrm{e}+01$

$1.44 \mathrm{e}+01$

$1.08+01$
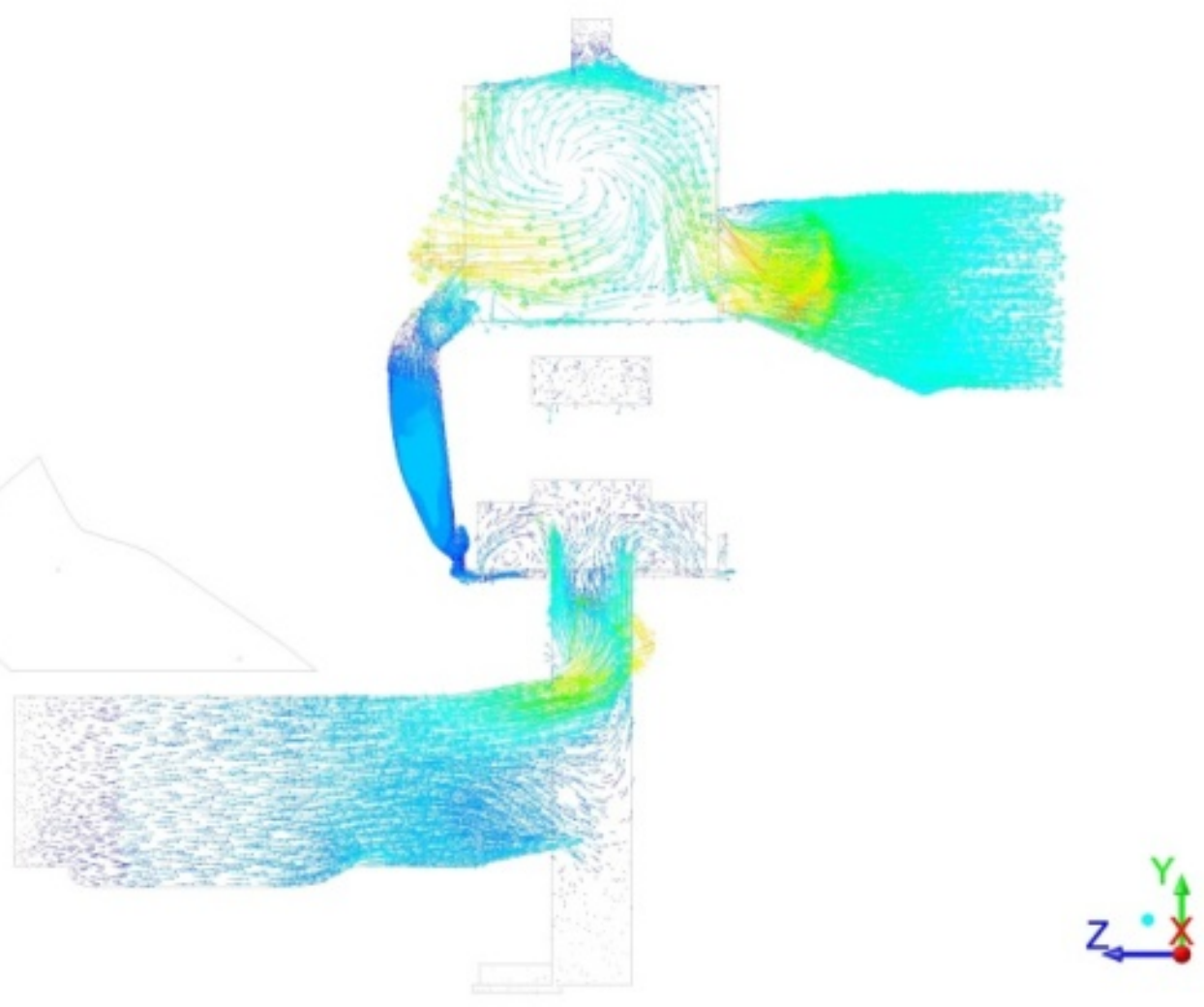

Velocity Vectors Colored By Velocity Magnitude $(\mathrm{m} / \mathrm{s}) \quad($ Time $=3.0000 \mathrm{e}-03)$

Oct 23, 2013 Crank Angle $=540.00$ (deg) ANSYS Fluent 14.5 (3d, dp, pbns, dynamesh, ske, transient)

Fig. (4). Field of distribution of velocity vectors in the longitudinal section.

\section{CONFLICT OF INTEREST}

The authors confirm that this article content has no conflict of interest.

\section{ACKNOWLEDGEMENTS}

This work was supported by the ministry of education and science of the Russian Federation in the framework of the implementation of the program of increasing the competitiveness of SSAU among the world's leading scientific and educational centers for 2013-2020 years.

\section{REFERENCES}

ANSYS Fluent User's Guide.
[2] V.V. Biryuk, A. A. Gorshkalev, D.V. Smorkalov and D.A. Uglanov, Modelling of thermal and gas-dynamic processes in a two-stroke piston engine with the help of FLUENT program. Samara: Samara State Aerospace University, 2011.

[23] V.V. Biryuk, A.A. and Gorshkalev, D.A, Calculation of thermal processes in the combustion chamber of a combustion engine with the help of FLUENT program. Uglanov - Samara: Samara State Aerospace University, 2011.

[4] V.V. Biryuk, A.A. Gorshkalev, S.S. Kayukov, and D.A. Uglanov, Modelling of thermal processes in the combustion chamber of a piston engine with direct injection. Samara: Samara State Aerospace University, 2012.

[5] M. Yu, Anisimov, V.V. Biryuk, A.A. Gorshkalev, S.S. Kayukov, and D.A. Uglanov, Creation of a finite element grid of a cylinderpiston group of a combustion engine. Samara: Samara State Aerospace University, 2013.

This is an open access article licensed under the terms of the Creative Commons Attribution Non-Commercial License (http://creativecommons.org/licenses/ by-nc/3.0/) which permits unrestricted, non-commercial use, distribution and reproduction in any medium, provided the work is properly cited. 